\title{
Application of Bifocal Concept to Dual Reflectarray Configurations for Multi-Beam Satellite Antennas in Ka-Band
}

\author{
Eduardo Martinez-de-Rioja , José A. Encinar , Antonio Pino , Borja Gonzalez-Valdes , \\ Carolina Tienda, Sean V. Hum , Giovanni Toso
}

\begin{abstract}
This contribution describes the design of a multibeam dual reflectarray antenna for operation in transmission in Ka-band (20 GHz). The bifocal design concept has been used to obtain an improved performance for the off-axis beams with respect to the single focused antenna. The required phase-shift distributions are initially obtained with the reflectarrays in parallel planes, and then adjusted to compensate the tilting of both reflectarrays in the final Cassegrain configuration. The simulated radiation patterns in the elevation and azimuth orthogonal planes have been calculated for the two beams generated by the focuses, and then the multi-beam performance of the antenna has been evaluated.
\end{abstract}

Index Terms-dual reflectarray, multi-beam antenna, bifocal design, satellite communications, Ka-band.

\section{INTRODUCTION}

Current communication satellite antennas in Ka-band are required to provide a high number of overlapping spot beams, typically more than 80 for a pan-European coverage [1]. This factor, combined with the deployment of frequency and polarization reuse schemes, leads to an increase in the users' data rates and the overall capacity of the network, enabling the provision of high speed broadband services in Ka-band.

Single and dual offset reflector antennas have been proposed for this type of multi-beam applications. In the case of dual reflectors, the offset configuration avoids blockage at the same time that offers more degrees of freedom than a single reflector antenna. In order to improve the scanning capabilities of the dual reflector, the bifocal design concept was introduced, first for a centered configuration [2], and then for its offset version [3]. The bifocal design provides a better performance for the off-axis beams in terms of gain and beam shaping, at the cost of a worse performance for the central beam.

Reflectarray antennas are able to generate independent beams in each polarization, provide high values of gain and radiation efficiency, and operate simultaneously at different frequencies [4]. These characteristics make them very interesting for multi-beam applications with frequency and polarization reuse, but firstly it is necessary to evaluate their performance for the generation of multiple spot beams.

In this paper, the authors present the design of a dual reflectarray antenna to produce multiple beams in transmission in Ka-band (20 GHz), applying the bifocal design concept for obtaining the required phase-shift distributions on both reflective surfaces. The results of the bifocal technique applied to reflectarrays were previously shown in [5] and [6], although the antenna presented a centered configuration and a small size. In this work, a dual offset reflectarray antenna for providing more than $47 \mathrm{dBi}$ gain at $20 \mathrm{GHz}$ is considered.

\section{BIFOCAL DESIGN PROCEDURE}

The classic bifocal synthesis can be applied to the design of reflectarray antennas, maintaining the same philosophy than in the case of reflectors. A ray tracing procedure has been implemented from the two focal points $\left(F_{1}\right.$ and $\left.F_{2}\right)$ to two scanned aperture planes. This technique alternates transmitted and received rays, in the same way that is described in [2]. However, a specific phase condition has to be considered on each reflectarray point, instead of applying the Snell's law of reflection. As shown in [5], the following expression relates the phase derivative on the reflectarray $(\partial \Phi / \partial \mathrm{r}$, where ' $r$ ' is the length variable along the reflectarray profile) with the angles of the incident $\left(\theta_{\mathrm{i}}\right)$ and reflected $\left(\theta_{\circ}\right)$ rays:

$$
\sin \theta_{o}-\sin \theta_{i}=\frac{\partial \Phi}{\partial r}
$$

The geometry of the dual reflectarray antenna is shown in Fig. 1. Originally, both reflectarrays are considered to be in parallel planes, in order to subsequently exploit the symmetry of the configuration. The initial conditions for the bifocal synthesis are: $\theta_{1}=1.5^{\circ}$ and $\theta_{2}=-1.5^{\circ}$ (angles of the radiated beams), $\mathrm{d}=20 \mathrm{~cm}$ (separation between $\mathrm{F}_{1}$ and $\mathrm{F}_{2}$ ), $\mathrm{L}_{1}=1 \mathrm{~m}$ (separation between the focuses and the sub-reflectarray), $\mathrm{L}_{2}=$ $1.5 \mathrm{~m}$ (separation between main and sub reflectarrays).

The bifocal synthesis originates two sets of points, one for the main reflectarray and one for the sub-reflectarray, with their 
corresponding phase derivatives. The phase derivatives are approximated by polynomial interpolation, and then, integrated to obtain the phase variation on each reflectarray. The resulting phase curves can be seen in Fig. 2.

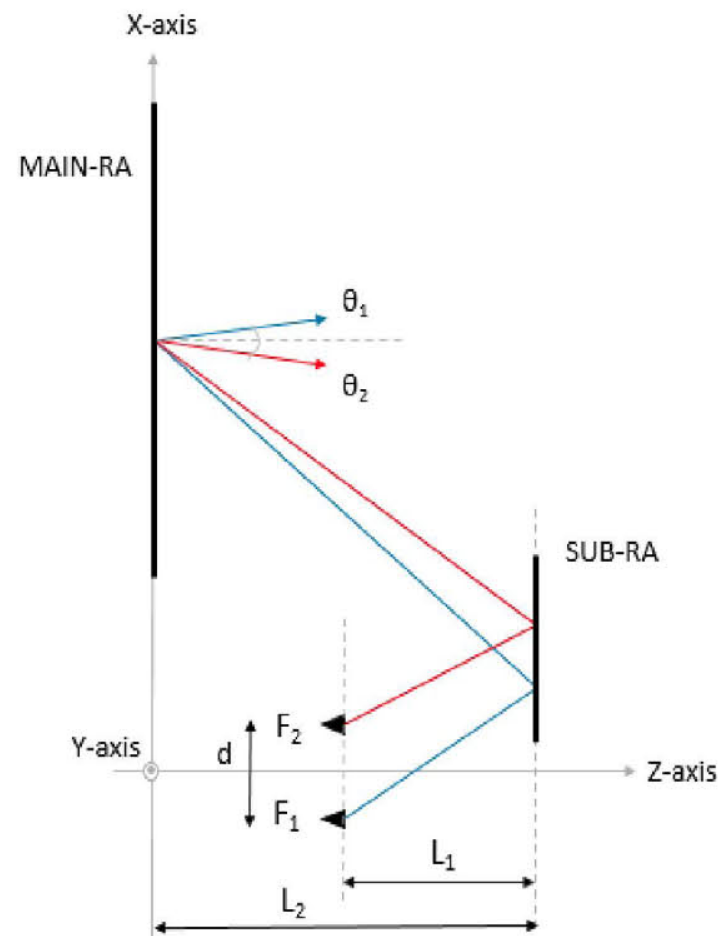

Fig. 1. Geometry of the bifocal dual reflectarray antenna

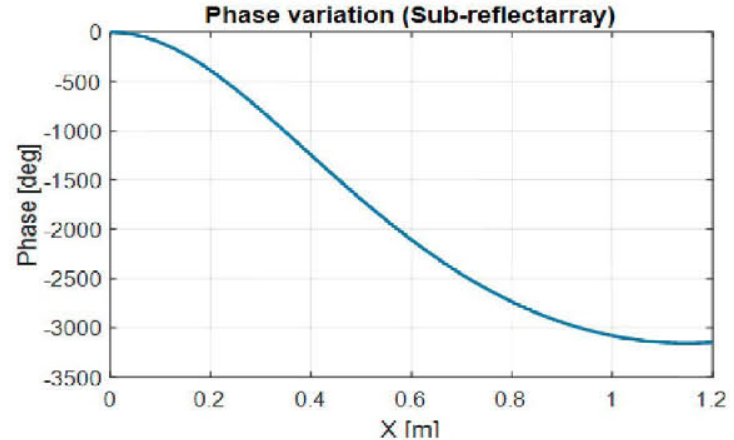

(a)



(b)

Fig. 2. Phase variation curves obtained by the bifocal technique for: (a) subreflectarray and (b) main reflectarray.
In order to obtain an offset configuration which minimizes blockage, the points from $\mathrm{x}=0.2 \mathrm{~m}$ to $\mathrm{x}=0.8 \mathrm{~m}$ have been chosen for constituting the sub-reflectarray ( $0.6-\mathrm{m}$ diameter), and the associated points from $\mathrm{x}=0.8 \mathrm{~m}$ to $\mathrm{x}=2.6 \mathrm{~m}$, for the main reflectarray (1.8-m diameter). However, the linear phase distributions shown in Fig. 2 only allow to collimate the beams in the XZ-plane. Therefore, the results of the bifocal synthesis have to be extended from 2D to 3D, so as to obtain a surface phase distribution on each reflectarray. In this case, taking advantage of the symmetry of the configuration with respect to Z-axis (see Fig. 1), both phase curves will be rotated in the XYplane around $Z$-axis. This process is also analogous to the one performed with reflectors [7], and results in a focal ring in the $\mathrm{XY}$-plane containing $\mathrm{F}_{1}$ and $\mathrm{F}_{2}$.

The main drawback of this design is that the phase-shift distributions present a high number of $360^{\circ}$ cycles. This makes difficult their practical implementation in a real reflectarray antenna, as the local periodicity approach, commonly used in the electromagnetic analysis of the reflectarray, assumes a smooth variation in phase between adjacent cells. The reasons for the high number of $360^{\circ}$ cycles are: the large size of both reflectarrays, the strict initial conditions of the bifocal synthesis, and the placement of both reflectarrays in parallel planes. Regarding the latter, this geometry was selected due to the possibility of applying rotational symmetry; however, a more natural configuration would present both reflectarrays tilted a certain angle with respect to $\mathrm{X}$-axis, trying to assimilate as much as possible to an equivalent Cassegrain reflector. As a consequence, the antenna geometry is modified in the way that is shown in Fig. 3, where main and sub reflectarrays are inclined $15^{\circ}$ and $10^{\circ}$, respectively (note that the focuses are also rotated with the sub-reflectarray).

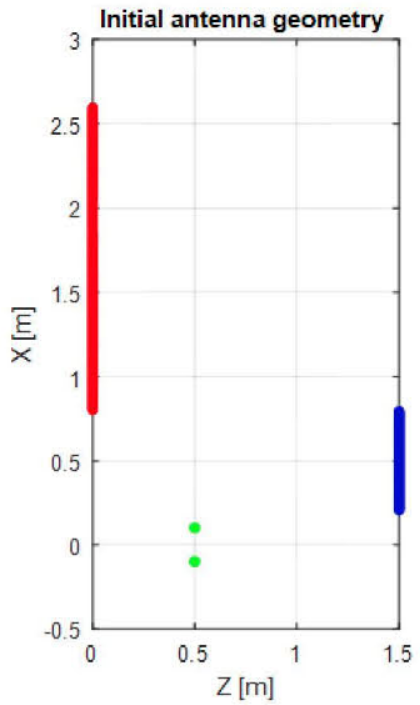

(a)

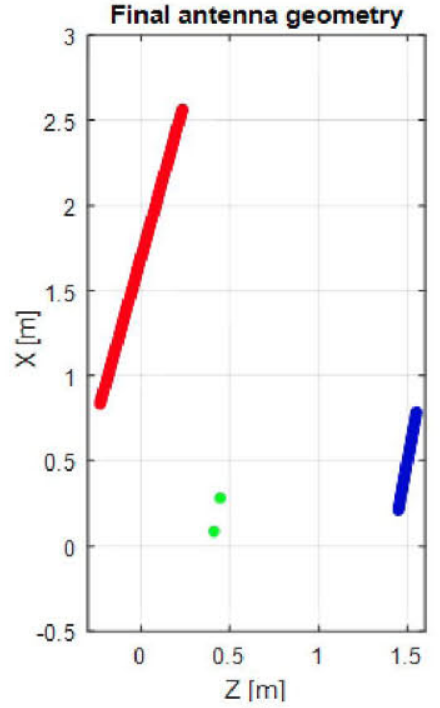

(b)
Fig. 3. Geometry of the dual reflectarray antenna: (a) initially, (b) after tilting both reflectarrays.

The tilting of both reflectarrays must be compensated in their phase-shift distributions, ensuring that the bifocal characteristic of the original design remains. A novel phase 
adjustment technique has been implemented, following a very similar procedure to the bifocal synthesis. The objective is to obtain a set of points in both reflectarrays with the appropriate phases that compensate the variations in the path length from the original configuration in parallel planes (Fig. 3a) to the new tilted antenna (Fig. 3b). The phase-shift distributions resulting from the application of this method are shown in Fig. 4. As can be seen, the phase presents a rather smooth variation, especially in the case of the sub-reflectarray. The most important result of this technique is that the antenna radiation patterns will present the same bifocal characteristic in the XZ-plane, while maintaining quite good results for the orthogonal plane, as a consequence of the previous rotation of the phase curves.

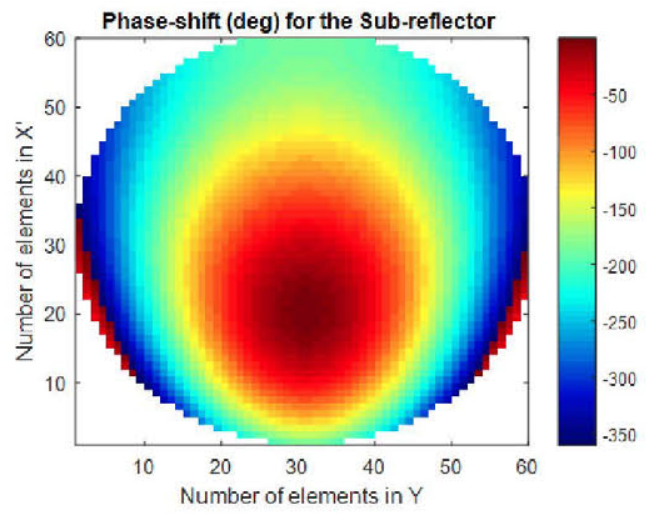

(a)



(b)

Fig. 4. Adjusted phase-shift distributions for: (a) sub-reflectarray and (b) main reflectarray.

\section{SiMULATION RESULTS}

The simulated radiation patterns for the original bifocal reflectarray antenna, with the two reflectarrays in parallel planes, are shown in Fig. 5. The results are presented at $20 \mathrm{GHz}$ (the transmission frequency in Ka-Band from a satellite), in the elevation and azimuth orthogonal planes. Note that the azimuth plane forms $1.5^{\circ}$ with respect to $\mathrm{Z}$-axis for the beam generated by $F_{1}$, and $-1.5^{\circ}$ for the beam generated by $F_{2}$, although they are superimposed in the same figure. As the design has been performed considering ideal phases, there are no cross-polar components of the radiated field. A gain of $49.6 \mathrm{~dB}$ is reached for the beam at $1.5^{\circ}$, and a $49.2 \mathrm{~dB}$ gain is attained for the beam at $-1.5^{\circ}$, both in the offset plane. The side-lobe levels are lower than $-20 \mathrm{~dB}$

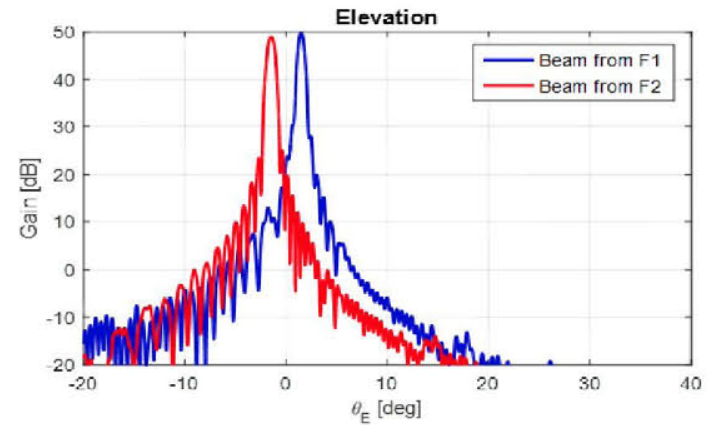

(a)

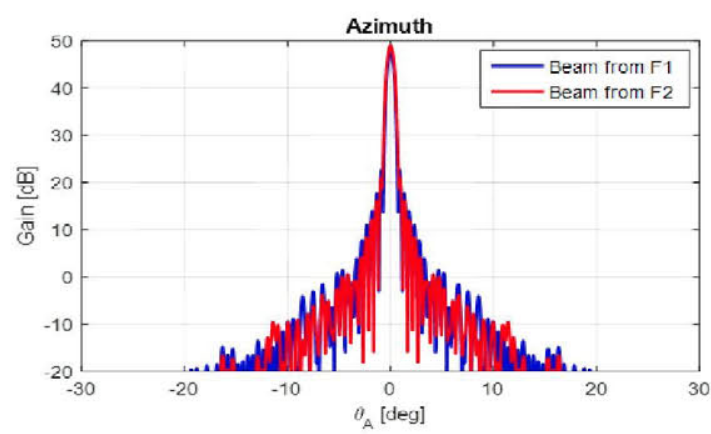

(b)

Fig. 5. Simulated radiation patterns for the dual reflectarray antenna after rotation of the phases.

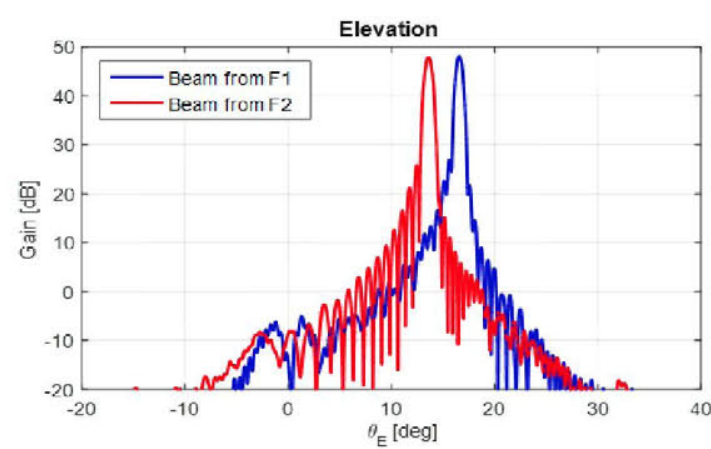

(a)

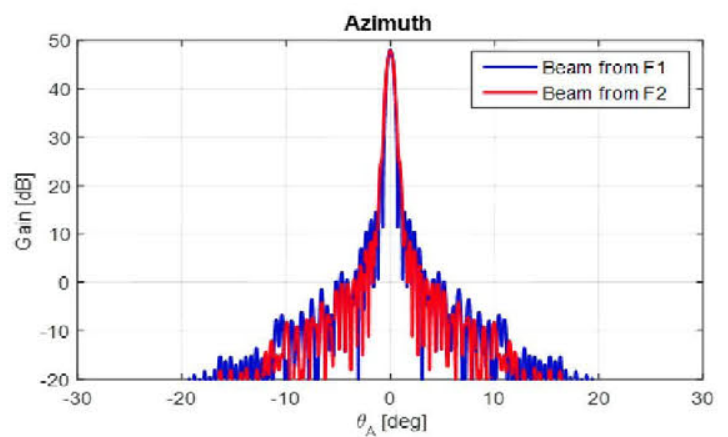

(b)

Fig. 6. Simulated radiation patterns for the dual reflectarray antenna after tilting both reflectarrays. 
The electromagnetic field radiated by the feed-horns is modelled by a cos- $q$ distribution, with $q=50$. The illumination level on the edges of the sub-reflectarray is close to $-12 \mathrm{~dB}$. The analysis of the dual reflectarray antenna has been carried out by using a specific homemade software tool, whose accuracy has been previously validated in other works involving the design, manufacturing and test of a dual reflectarray demonstrator [8].

Figure 6 shows the radiation patterns of the final dual reflectarray antenna. As can be seen, there is a small loss in gain, about $1.4 \mathrm{~dB}$, which is mainly due to the inclination of the main reflectarray. This factor causes a slight reduction in the effective aperture of the antenna, as well as a beam displacement from the initial directions at $\pm 1.5^{\circ}$ to the final directions at $15^{\circ} \pm 1 \cdot 5^{\circ}$. Despite this fact, the beam shaping and side-lobe levels are conserved.

After the calculation of the radiation patterns for the two feeds placed at the focal points, $F_{1}$ and $F_{2}$, additional feeds are considered in order to evaluate the multi-beam performance of the antenna. First, an array of nine horns is placed on the XZplane, so that the second and the eighth feeds correspond to the focal points $F_{1}$ and $F_{2}$. The simulated patterns in the elevation plane for the nine beams are shown in Fig. 7. As can be seen, the adjacent beams are separated $0.5^{\circ}$, the gain varies from $47.65 \mathrm{~dB}$ to $48.2 \mathrm{~dB}$, and side-lobe levels are around $-20 \mathrm{~dB}$. Moreover, the performance of the antenna has been also evaluated for the beams in the orthogonal plane to the XZ (out of the plane where the bifocal synthesis was performed). The pattern contours of $47.5 \mathrm{~dB}$ and $45 \mathrm{~dB}$ are shown in Fig. 8 for the five central beams in the XZ-plane and the five central beams in the orthogonal direction.

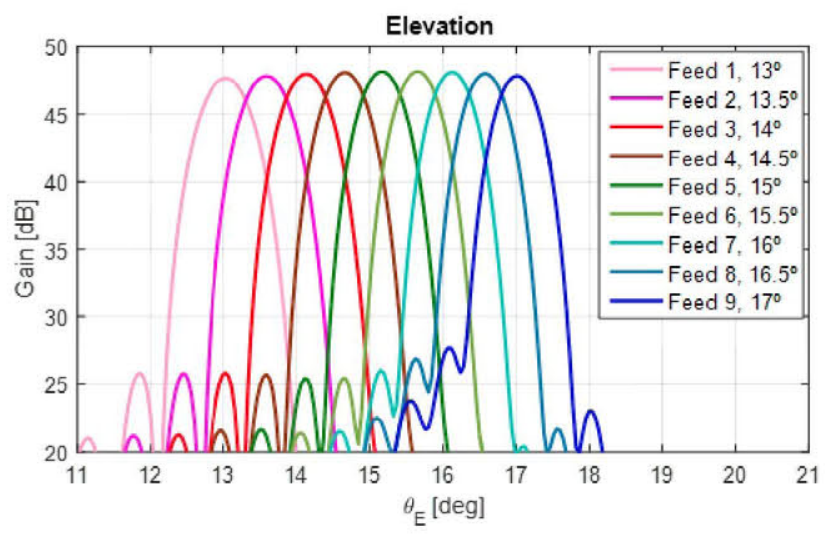

Fig. 7. Simulated radiation patterns for the dual reflectarray antenna considering a linear array of 9 feeds in the XZ-plane.

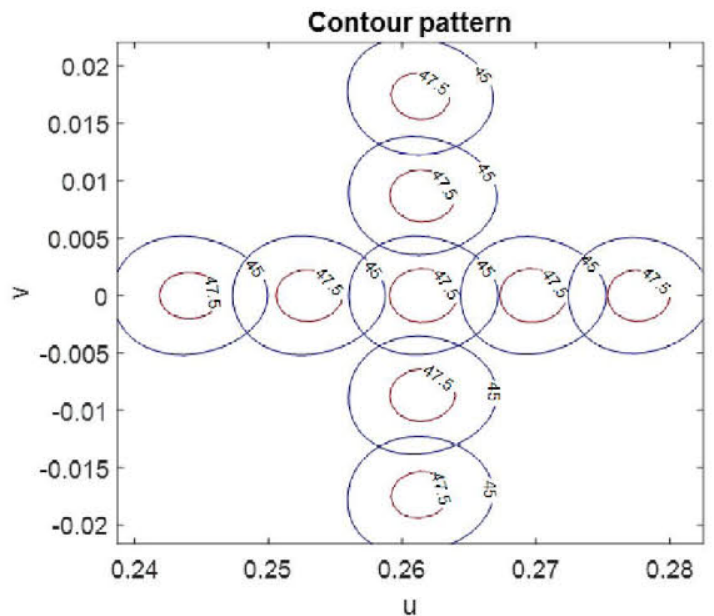

Fig. 8. Radiation pattern contours for the five central beams in the XZ-plane and the five central beams in the orthogonal plane.

\section{CONCLUSIONS}

These results show the potential of reflectarray antennas for multiple beam generation, applying the bifocal design concept for obtaining improved performance for the off-axis beams. The next step will involve the implementation of a more detailed design with real specifications of multi-beam antenna systems, considering also non-ideal reflectarray elements.

\section{ACKNOWLEDGMENT}

This work has been supported by the Spanish Ministry of Economy and Competitiveness under the project TEC201343345-P and by the European Space Agency (ESA) under contract 4000117113/16/NL/AF.

\section{REFERENCES}

[1] M. Schneider, C. Hartwanger, H. Wolf, "Antennas for multiple spot beams satellites", CEAS Space Journal, Vol. 2, pp. 59-66, Dec. 2011.

[2] B. L. Rao, "Bifocal Dual Reflector Antenna", IEEE Trans. on Antennas and Propag., Vol. 22, No. 9, pp. 711-714, Sept. 1974.

[3] C. M. Rappaport, "An Offset Bifocal Reflector Antenna Design for WideAngle Beam Scanning", IEEE Trans. on Antennas and Propag., Vol. 32, No. 11, pp. 1196-1204, Nov. 1984.

[4] J. Huang and J. A. Encinar, "Reflectarray Antennas", IEEE Press/Wiley, Piscataway, New Jersey, 2008.

[5] W. Menzel, M. Al-Tikriti, R. Leberer, "A 76-GHz multiple-beam planar reflector antenna", Proc. of 32nd European Microwave Conference, Milan, Italy, Sept. 2002.

[6] J. G. Cuevas, C. Tienda, J. A. Encinar and G. Krieger, "Principle of Bifocal Antennas Implemented in a Dual Reflectarray Configuration", $9^{\text {th }}$ European Conference on Antennas and Propagation, Lisbon, Portugal, 12-17 April 2015.

[7] A. Garcia-Pino, N. Llombart, B. Gonzalez-Valdes and O. RubinosLopez, "A bifocal ellipsoidal gregorian reflector system for THz imaging applications", IEEE Trans. on Antennas and Propag., Vol. 60, No. 9, pp. 4119-4129, Sept. 2012.

[8] C. Tienda, J.A. Encinar, M. Arrebola, M. Barba and E. Carrasco, "Design manufacturing and test of a dual-reflectarray antenna with improved bandwidth and reduced cross-polarization", IEEE Trans. on Antennas and Propag., Vol. 61, No. 3, pp. 1180-1190, March 2013. 